Article

\title{
Analysis of Lower Hand Wrist Flexion and Twist of the Mallet Head in a Croquet Shot
}

\author{
Jenny Clarke \\ School of Health Sciences, University of Canterbury, Christchurch 8140, New Zealand; \\ jenny.clarke@canterbury.ac.nz
}

Received: 6 May 2020; Accepted: 15 June 2020; Published: 18 June 2020

\begin{abstract}
This study investigated the relationship between wrist flexion and the dynamics of the swing of a croquet mallet. Twenty-seven subjects participated in a study which used 3D motion capture equipment along with high-speed and high-definition video to determine if there is a correlation between the lateral twist of a croquet player's swing and the flexion of the wrist during that swing. The study found a significant correlation between the amount of flexion of the wrist from the start of the stroke to the top of the backswing and the twist of the mallet head at the top of the backswing $(r=0.330 ; p<0.01)$. The methodology and findings are relevant to all sports where minimising wrist flexion is favourable for improving the consistency of stroke-making (e.g., golf putting, darts and snooker). Additionally, reducing wrist motion in stroke-making may reduce the incidence of wrist pain and injury in croquet. This second point provides further encouragement for attempting to reduce the amount of wrist flexion during croquet swings.
\end{abstract}

Keywords: wrist injury; motion capture; croquet; technique analysis; overuse

\section{Introduction}

Croquet is a game played by two or four players where the aim is to score points by hitting a ball through a series of hoops in a predefined order using a mallet [1]. The mallet has a shaft which is typically $85-100 \mathrm{~cm}$ long, ending in a rectangular head which is $22-30 \mathrm{~cm}$ long. The mallet is made of wood, or another material which does not give a playing advantage over wood. The ends ("end faces") of the mallet head, which are used to strike croquet balls, are parallel, and the overall mallet weight is $1-1.5 \mathrm{~kg}$.

The mallet is held vertically, with the mallet head initially resting between the player's ankles. The player stands approximately $30 \mathrm{~cm}$ behind the ball they wish to strike, pointing in the direction of aim. Hips and knees should be slightly flexed, shoulders relaxed and fully extended and the elbows slightly flexed. Both hands grasp the mallet at the top of the shaft. The mallet is initially drawn back between the player's ankles (the "backswing"), then forward to strike the ball. For the purposes of this study, the shot is defined as starting with the mallet resting between the feet.

Like other stationary-ball sports, where the ball is struck with the distal end of an implement (e.g., golf and snooker), the game of croquet requires accuracy and consistency, rewarding a controlled and repeatable swing. Similar to the putting stroke in golf, the croquet mallet is held approximately vertically and gripped near the top, striking a ball which is initially stationary on the ground. Unlike golf (Rule 10.1c, [2]) croquet is played with a mallet which is swung, for the vast majority of modern players, between the player's legs, with the player facing the direction they wish to hit the ball.

If the mallet is drawn backward and then forward straight along the line of aim, contact with the ball at any point on the mallet's forward trajectory will cause the ball to be propelled in the desired direction. By contrast, a stroke where the head of the mallet twists from left to right, or from right to left, as it is swung, requires precise timing to drive the ball accurately towards its target. Twisting motions mean that the player needs to have fully corrected-and not overcorrected-the alignment of the mallet 
immediately before impact with the croquet ball. Many international level players twist their mallet appreciably during the backswing, for example a five-time world champion has an approximately 10-degree twist in his backswing, and a two-time New Zealand Open champion has a highly exaggerated twist of around 20-25 degrees. These players, and several other top players, however, have noted the difficulty of recovering their accuracy during a "bad patch" of play. This includes incidences of the "yips", firmly established in golf [3], and also a problem for many croquet players. Furthermore, beginner and club level players who do not have a straight swing struggle to develop the ability to consistently hit a croquet ball in the desired direction. In golf, techniques such as various wrist-locking devices $[4,5]$, and motion capture and wearable technology analyses [6,7] have been used to help understand and correct wrist rotation and swing inconsistencies. It is therefore of interest to better understand mechanisms which may cause the croquet mallet to twist, or to veer off path, in order to optimise competitive performance.

An additional concern is the occurrence of wrist discomfort and diagnosed injuries among croquet players [8]. Of 214 survey participants, $36 \%$ reported at least one injury to the "hand, wrist or forearm" caused by striking a croquet ball [8] (page 219). While no specific research has been conducted on the epidemiology of wrist injuries in croquet, a vast body of research demonstrates the prevalence of injury in racquet sports where the wrist is repeatedly forcefully flexed and extended with movement across gliding joints of the wrist, in addition to striking forces which affect the wrist during contact between the striking implement and the ball $[9,10]$. At $454 \pm 7$ grams, a croquet ball is significantly heavier than most balls struck in racquet sports.

To date, there have been no kinematic analyses undertaken of the sport of croquet. As a result, very little is known about the dynamics of a croquet swing. One injury surveillance study has been carried out [8], and otherwise the only published studies about croquet relate to coaching pedagogy and tournament management. This article attempts to begin to address the absence of research into this sport.

Reducing the amount of movement in the wrist during a croquet swing, particularly at the moment of impact of the mallet with the ball, is one mechanism to address wrist injuries. This is particularly relevant when considering the age demographic of typical croquet players. The 7500 croquet players who belong to the UK Croquet Association, for example, have a median age of 70 years old, with an average age of players newly taking up the sport of 66 [11].

The aim of this study is to investigate the relationship between wrist flexion and rotation of the croquet mallet head about the mallet's shaft. Figure 1 shows the standard croquet grip, where the "lower wrist" is defined by the hand in the inferior position. In particular, we explore flexion of the lower wrist. At the starting position, that wrist is already in ulnar deviation (flexed towards the ulna); therefore, any changes from this position tend to forcefully move the wrist towards the limits of its possible range of motion in that direction.

We hypothesised that lateral deviation of the mallet head (i.e., twisting about the long axis through the shaft of a croquet mallet) would be correlated with flexion of the lower wrist. 


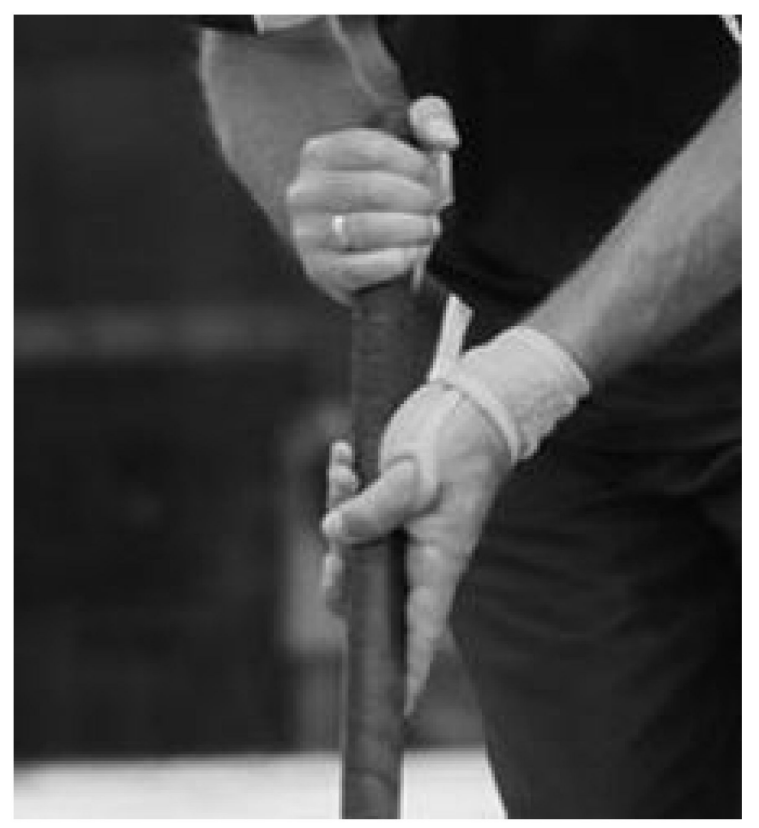

Figure 1. The croquet "standard grip", with the knuckles of the upper hand and the palm of the lower hand facing forward. Both hands are relatively close together. The "lower wrist" is defined as the wrist on the inferior hand in this position. The wrist support shown is used by an injured player and is not standard equipment.

\section{Methods}

Twenty-seven croquet players (19 male and 8 female players) from the Canterbury Croquet Association (CCA) who satisfied the criteria of using a standard grip and were available during the testing period participated in the study. The participants ranged in age from 21 to 85 years old (mean $=64.6$ years; standard deviation $=9.1$ years), with a mean of 12.6 years playing experience (standard deviation $=4.2$ years). All participants were fully briefed on the procedures, data collection and handling techniques to be used and all signed an informed consent document.

All of the players used the standard grip (see Figure 1), with hands either close together or slightly overlapping. A BTS Bioengineering SMART-D 140 marker-based motion capture system with six infrared DX100 cameras was used [12]. Thirteen reflective markers were placed on the players and equipment. The critical markers on the players were placed on the tendon of the abductor pollicis longus where it crosses the wrist joint, on the head of the second metacarpal on the posterior of each hand, and on the lateral epicondyle of the humerus of each arm. Additional markers were placed at the front and back of the head of the mallet and at the top of the shaft of the mallet. Data were collected at $100 \mathrm{~Hz}$ with the data acquisition system calibrated to a precision of $\pm 0.5 \mathrm{~mm}$. This reconstruction accuracy is consistent with the manufacturer's product specifications.

Each participant took 10 shots hitting a croquet ball with their own mallet in their usual stance and style at a stationary croquet ball which was $4.5 \mathrm{~m}$ from the striker's ball. This distance was chosen as it optimised the use of available laboratory space and to encourage the players to strike their ball at moderate pace. The five shots for which the system reconstructed the marker movements with the most complete tracks were used for each player. The average flexion of the wrist from the starting point of the swing to the peak of the backswing was determined, as well as the rotation of the mallet about its shaft during each shot.

Marker data were captured using the BTS SMART Motion Capture System [12]. The vectors, defined by the points marking the two ends of the mallet head, were projected onto an XY plane parallel to the ground, with the twist angle measured in this plane. Wrist flexion was measured for the bottom hand relative to the starting position. The marker placements are shown in Figure 2. The 3D angle 
between markers 1, 2 and 3 was used to quantify wrist flexion. Mallet rotation was determined from the change in angle of the vector from marker 4 to marker 5 , projected onto the ground, from the starting position of the swing to the top of the backswing. The wrist angle referred to here is therefore the angle of the lower wrist from the participant holding the mallet with a "standard grip", measured between the $5^{\text {th }}$ metacarpal and the ulnar surface of the forearm. While this angular deviation can involve some radioulnar deviation, the measurements taken in this study were simplified to measure only the magnitude of the metacarpal-ulnar angular changes and not the specific alignment of the wrist.

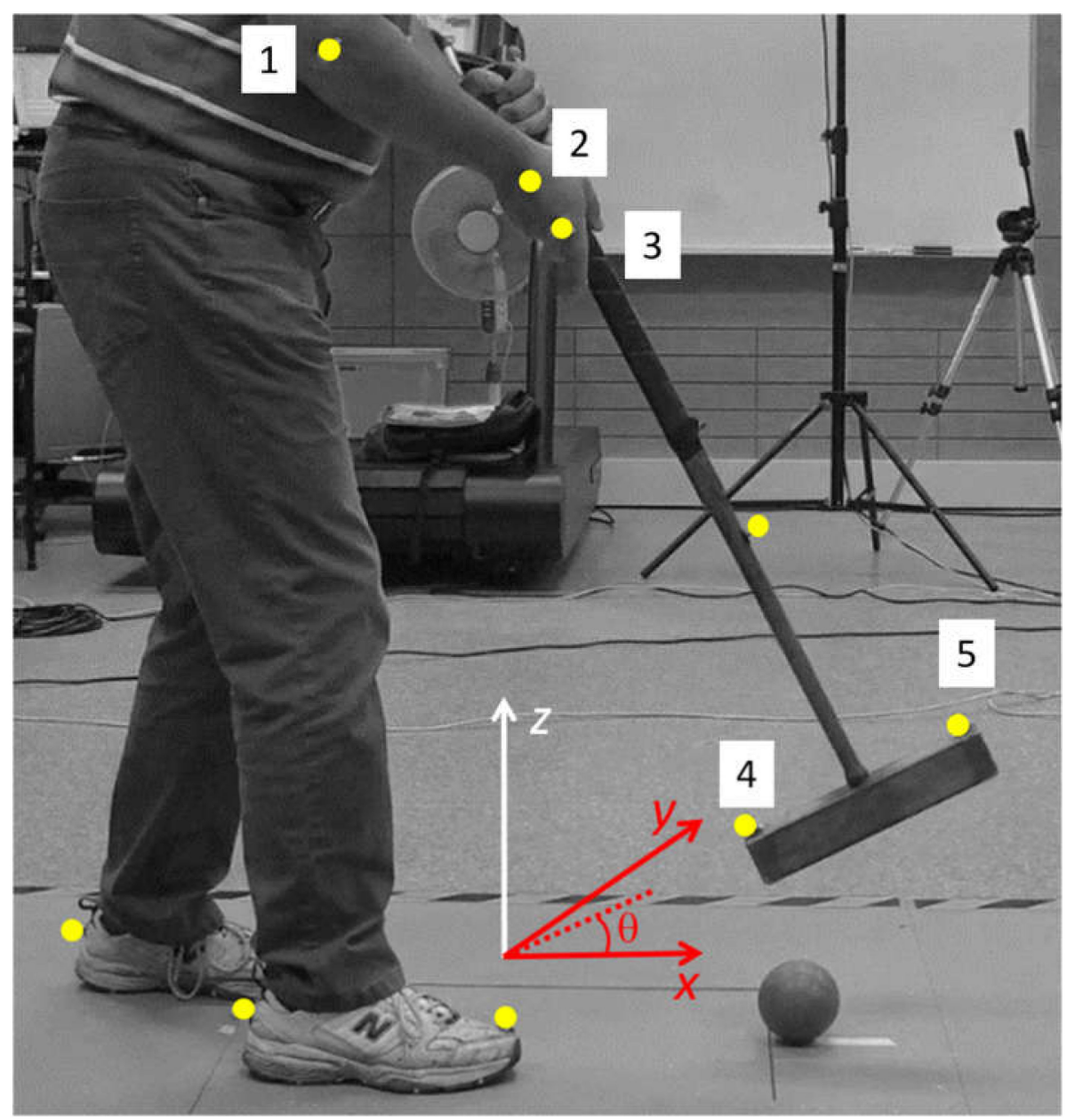

Figure 2. Marker placements shown on study participant with yellow dots. Markers labelled 1, 2 and 3 define the wrist angle used in the study, while markers 4 and 5 are used to quantify the mallet direction during croquet stroke. The axis system shown indicates the xy plane on the ground, with the vector between markers labelled 4 and 5 projected onto the ground and the mallet angle $\theta$ defined on the xy projection as shown. Image of participant used with permission.

IBM SPSS statistics package Version 23.0 [13] was used to compare the angle of maximum wrist flexion from the starting position with the angle of maximum mallet deviation, as defined above. A Pearson correlation was used to test the null hypothesis (no relationship between wrist flexion during the swing and lateral deviation of the mallet during the swing). Significance to reject the null hypothesis was set at $p<0.05$. 


\section{Results}

The average flexion in wrist angle from the starting position was $7.1 \pm 3.9$ degrees, ranging from virtually no bending to a maximum of 19 degrees. Rotation of the mallet head about the shaft varied from 0 to 15.4 degrees, with an average twist angle of $3.6 \pm 3.1$ degrees.

A scatterplot of wrist angle change compared to mallet deviation is shown in Figure 3 with a fit line superimposed. A Pearson correlation test showed a statistically significant correlation and moderate relationship between wrist flexion and mallet twist $(p<0.01, r=0.33)$. The linear regression had an $\mathrm{R}^{2}$ value of 0.109 .

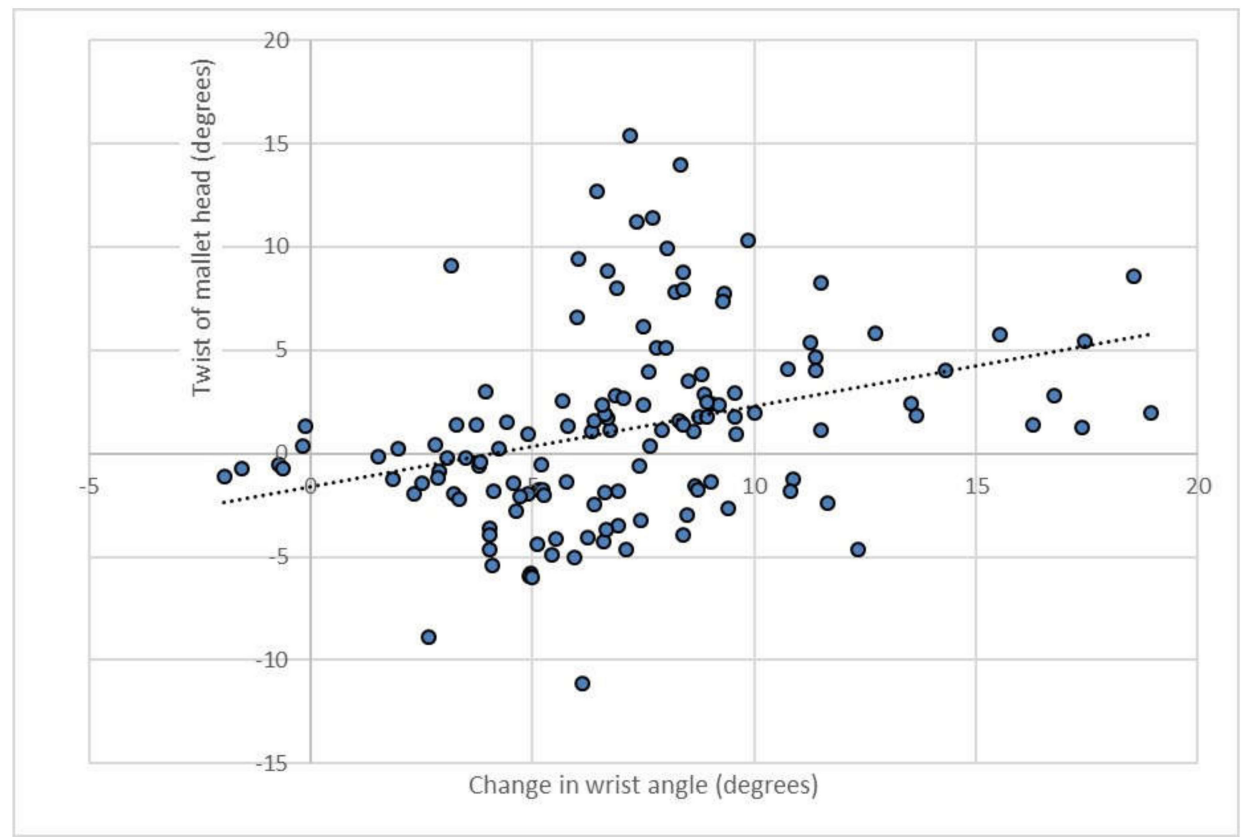

Figure 3. Scatterplot showing comparison of change of wrist angle from address to top of backswing and angle of twist of mallet during a croquet shot. Positive wrist angle change represents an increase in the angle between the thumb and the radial surface of the forearm, while positive mallet twist refers to rotation of the front surface of the mallet head towards the right, for a right-handed player. Each data point represents an individual data point (one trial of one participant).

\section{Discussion}

The study found a significant relationship between flexion of the lower wrist of a standard grip croquet player, between the start of the backswing and the peak of the backswing, and rotation of the mallet about its shaft.

Numerous patented golf inventions seek to minimize or eradicate wrist flexion to create a consistent putting stroke. There are few publications relating to the sport of croquet; however, the croquet swing has strong similarities to the golf putting stroke, from which it is reasonable to suspect that reducing wrist flexion may improve consistency in croquet. Consistency in this context means having a longer period of time when swinging the mallet where the mallet head is pointing squarely towards the target. In addition, a significant proportion of croquet injuries involve wrist pain. Reducing wrist flexion during stroke-making should reduce torsional impacts on the wrist and reduce the occurrence of wrist pain and injuries in croquet players. This suggests a strong possibility that a mechanism generating a crooked backswing is lower wrist flexion.

An important rationale for this study was the author's field observations over 20 years of experience in the sport. There is a current lack of studies on the sport of croquet. In particular, there is no previous research published on the kinematics of the croquet swing. It is important that these 
studies are carried out because croquet is a sport which is increasing in popularity worldwide, with more people playing croquet and a greater number of elite international events. Additionally, with the majority of croquet players being over 65 years old, it is particularly important to understand the risk factors for injury to enable people to continue to be active for longer. This study is a first step to improving the understanding of the croquet swing and exploring a possible mechanism for reducing wrist pain.

Further research in this area could include interventions to directly reduce the magnitude of twist of the mallet during the swing. Two possible simple interventions are practising with a mirror to purposefully reduce mallet twist, or psychological techniques such as deliberately trying to twist the mallet in the opposite direction to the natural deviation.

It would also be interesting to study interventions to directly reduce wrist flexion, to discover if these produce a straighter swing. Practice routines, such as visualisation of swinging a mallet back and forth with the wrist staying in a fixed angle of flexion, then practising swinging the mallet without a ball to hit but focusing on keeping the wrist held at fixed flexion, are worth investigating. Further, golf-like wrist supports or strapping, which bind the wrist into a relatively fixed position could be used to physically reduce wrist motion. It is notable that strapping and physical devices which limit wrist motion are not forbidden by the Laws of Association Croquet, or the Rules of Golf Croquet [14], therefore suggesting that if these physical mechanisms are effective in reducing mallet twist, they could be incorporated into the normal equipment of croquet players. While also not forbidden in golf, it is necessary to have a full range of wrist flexion for shots played away from the putting green. Croquet warm-up and training aids, such as the "Bamford Swing Trainer" [15], which physically prevents the mallet from twisting through the use of rigid guide panels, are also worthy of investigation. Longitudinal analyses of players who have undergone interventions, compared against a control sample, would also be useful in investigating the effect of reducing wrist flexion on controlling wrist pain and reducing the occurrence of wrist injuries.

Programmes involving physical practice and mental practice techniques, such as visualization, would need to be controlled for general improvement due to practice alone. Any such intervention should be assessed both immediately after the techniques have been applied and again some weeks later, to assess both the short-term efficacy of the technique and the long-term response.

Results from the present study are relevant to ball sports where accuracy in stroke-making is optimised with limited wrist flexion and the primary upper limb movement being dominated by larger muscles across the shoulder and elbow joints, such as golf, squash and cricket $[10,16]$. Additionally, it is noteworthy that wrist injuries through both impact and overuse of the gliding joints in the wrist are prevalent in the aforementioned sports $[9,10]$. Both the methodology for the data collection and the hypothesis are important to sports where wrist engagement is important, as well as sports where wrist injuries are common.

\section{Conclusions}

This study suggests that a relationship could exist between flexion of the wrist during a croquet shot and rotation of the mallet about a longitudinal axis through its shaft. This relationship provides a possibility to train croquet players to improve their technique through working to minimise wrist movement.

In addition, one of the few publications to date relating to croquet has identified wrist injury as a significant affliction of croquet players. Reducing wrist movement during a croquet shot could reduce torsional forces on the wrist, thereby reducing the occurrence of repeated impact injuries.

Funding: This research received no external funding.

Acknowledgments: The author is particularly grateful for the assistance of University of Canterbury Sport Science technician Gavin Blackwell for all aspects of data collection, and members of the Canterbury Croquet Association who volunteered their time to participate in this study.

Conflicts of Interest: The author declares no conflict of interest. 


\section{References}

1. Croquet Association The Laws of Association Croquet-6th Edition. Amended 2008. Available online: https://www.croquet.org.uk/?p=games/association/laws/6th/laws6th (accessed on 30 March 2019).

2. The R\&A Rules of Golf. Available online: https://www.randa.org/en/rog/2019/pages/the-rules-of-golf (accessed on 30 March 2019).

3. Philippen, P.B.; Legler, A.; Land, W.M.; Schuetz, C.; Schack, T. Diagnosing and measuring the yips in golf putting: A kinematic description of the involuntary movement component that is the yips. Sport Exerc. Perform. Psychol. 2014, 3, 149. [CrossRef]

4. Dalbo, L. Golf Swing Forearm/Wrist Positioner. U.S. Patent 5,501,464, 26 March 1996.

5. Leonard, C. Wrist Locking Golf Training Device. U.S. Patent 6,656,056, 2 December 2003.

6. Sim, M.; Kim, J.-U. Differences between experts and novices in kinematics and accuracy of golf putting. Hum. Mov. Sci. 2010, 29, 932-946. [CrossRef] [PubMed]

7. Ghasemzadeh,H.; Loseu, V.; Guenterberg, E.; Jafari, R. Sport training using body sensor networks: A statistical approach to measure wrist rotation for golf swing. In Proceedings of the Fourth International Conference on Body Area Networks ICST, Los Angeles, CA, USA, 1-3 April 2009; p. 2.

8. Appleton, D. A survey of croquet injuries. J. R. Soc. Med. 1997, 90, 218-220. [CrossRef] [PubMed]

9. Osterman, A.; Moskow, L.; Low, D. Soft-tissue injuries of the hand and wrist in racquet sports. Clin. Sports Med. 1988, 7, 329-348. [PubMed]

10. Rettig, A.C. Athletic injuries of the wrist and hand: Part II: Overuse injuries of the wrist and traumatic injuries to the hand. Am. J. Sports Med. 2004, 32, 262-273. [CrossRef] [PubMed]

11. Carter, K. A Survey of Croquet Players; Profundus Consulting Ltd: London, UK, 2017; p. 87.

12. BTS Bioengineering BTS SMART-DX. Available online: http://www.btsbioengineering.com (accessed on 30 March 2020).

13. IBM Corp. IBM SPSS Statistics for Windows, Version 23.0; IBM Corp: Armonk, NY, USA, 2015.

14. World Croquet Federation WCF Sports Regulations. Available online: https://www.worldcroquet.org.uk/ documents/Governance/SportsRegs/WCFSportsRegulations.pdf (accessed on 30 March 2020).

15. Hobbs, J. The Bamford Swing Trainer. 2010. Available online: http://www.oxfordcroquet.com/equip/ swingtrainer/index.asp (accessed on 9 June 2020).

16. Howse, C.M. Wrist injuries in sport. Sports Med. 1994, 17, 163-175. [CrossRef] [PubMed] 